\title{
Effect of random magnetic field on active-sterile neutrino conversion in the supernova core
}

\author{
Sarira Sahu* and Vishnu M. Bannur \\ Institute For Plasma Research, Bhat, Gandhinagar-382 428, India

\begin{abstract}
The active-sterile neutrino conversion is studied for neutrino propagating in the axial
\end{abstract} \\ potential generated by magnetised electron plasma in the supernova medium. We consider \\ the effect of random magnetic field $B_{r m s}$ on the average neutrino conversion probability. We \\ obtained the constraint on $\Delta m^{2}$ and $\sin ^{2} 2 \theta$ for different strength of the random magnetic \\ fields, by considering the positive definiteness of the average neutrino conversion probability \\ inside the supernova core. Our calculation shows that, $B_{r m s} \lesssim 0.63 \times 10^{14}$ Gauss is preferable \\ so that small values of $\Delta m^{2}$ can not be excluded.
}

\section{INTRODUCTION}

The COBE measurement of the cosmic microwave background temperature anisotropies on large scale [1], hints for the existence of a hot dark matter (HDM) component of $30 \%$ of the total mass density [2]. Also the recent results of solar neutrino, atmospheric neutrino and the LSND result, hints for the neutrino oscillation. All these can not be explained with the three generation neutrino flavor mixing scheme. Thus the speculation is that, there is a fourth generation low mass sterile neutrino $\nu_{s}$ which will mix with the standard model neutrinos. This neutrino has to be sterile with respect to electroweak interactions so that, it can not be detected directly through experimental searches. This postulate of a fourth generation lightsterile neutrino can explain simultaneously the dark matter, solar neutrino and atmospheric problems [3 6 . The most stringent constraints for the neutrino mass matrix including a sterile neutrino species are obtained from the nucleosynthesis bound on the maximum number of extra neutrino species that can reach thermal equilibrium before nucleosynthesis and change the primordially produced helium abundance [7].

Neutrino propagation in the magnetised medium has interesting consequences in the astrophysical and cosmological scenarios. Large scale magnetic fields in the early universe hot plasma and in the core of the supernova can effect the neutrino conversion [8]. It has been shown that random magnetic fields can strongly influence neutrino conversion rates and this could have important implications, especially in the case of conversion involving a light sterile neutrino [9,10]. The effect of active-sterile neutrino conversions in a supernova has also been discussed, both in the case where no magnetic field is present, as well as in the presence of random magnetic field [10] as large as $10^{16}$ Gauss.

The dispersion relation for neutrino propagating in a magnetised medium is different from the vacuum one. In the magnetised medium neutrino acquires an axial potential which is proportional to the scalar product of the neutrino momentum and the magnetic field vector (k.B). The effect of axial potential on neutrino propagation in media with regular and/or random magnetic fields are considered in the literature [10 13]. For random magnetic fields case Semikoz and Valle 13] have show that, the neutrino conversion is aperiodic in nature. The effect of axial potential on active-sterile neutrino conversion has also been considered in supernova and sun 10,14. It was shown by Kusenko and Segre recently that, the polarisation effects of the supernova medium lead to the explanation of the birth velocity of pulsars 15$]$. 
In this paper we have considered the effect of axial potential on neutrino propagation in the supernova medium in the presence of random magnetic fields. We calculate the average neutrino conversion probability $\mathcal{P}$, for the process $\nu_{e} \rightarrow \nu_{s}$ using two different approximations (method-I and II). method-I shows that, for both $\Gamma_{\|}>\omega_{f}$ and $\Gamma_{\|}<\omega_{f}$ ( $\Gamma_{\|}$is the longitudinal damping parameter) one can obtain finite conversion probability for $\nu_{e} \rightarrow \nu_{s}$. On the other hand for the positive definiteness of the conversion probability in method-II, only $\Gamma_{\|}<\omega_{f}$ is possible. Using this positive definiteness condition of the average conversion

probability inside a supernova core, we have showed that $B_{r m s} \lesssim 0.63 \times 10^{14}$ Gauss is preferred, because large values of $B_{r m s}$ exclude small values of $\Delta m^{2}$, which are not supported by present experiments.

The paper is organised as follows: in Sec. II we calculate the average neutrino conversion probability using two different methods in the presence of a randomly fluctuating magnetic field. The comparison of both the methods are undertaken in Sec. III and necessary condition for the positive definiteness of the average conversion probability for the process $\nu_{e} \rightarrow \nu_{s}$ is obtained in the second method. Using the necessary condition for active-sterile neutrino conversion inside a supernova core, we put constraint on the parameters $\Delta m^{2}$ and $\sin ^{2} 2 \theta$ for different values of the random magnetic field in Sec. IV and a short conclusion is drawn in Sec. V.

\section{NEUTRINO PROPAGATION IN THE MAGNETISED MEDIUM}

\section{A. method - I}

The evolution equation for a system of two neutrinos $\nu_{a}$ and $\nu_{b}$, where $\nu_{a}$ is the active one and $\nu_{b}$ is active/sterile one is given by

$$
i \frac{d}{d t}\left(\begin{array}{c}
\nu_{a} \\
\nu_{b}
\end{array}\right)=\left(\begin{array}{cc}
H_{a a}(t) & H_{a b}(t) \\
H_{b a}(t) & H_{b b}(t)
\end{array}\right)\left(\begin{array}{c}
\nu_{a} \\
\nu_{b}
\end{array}\right)
$$

where the quantity $H$ is in general the potential for the neutrino in the medium which we will discuss soon. Let us define the functions $R=\operatorname{Re}\left(\left\langle\nu_{a}^{*} \nu_{b}\right\rangle\right)$ and $I=\operatorname{Im}\left(\left\langle\nu_{a}^{*} \nu_{b}\right\rangle\right)$. Then using these in Eq.(1) we obtain

$$
\dot{R}(t)=-H_{d}(t) I(t), \quad \dot{I}(t)=H_{a b}(t)(2 P(t)-1)+H_{d} R(t),
$$

and

$$
\dot{P}(t)=-2 H_{a b}(t) I(t)
$$

where the function $P(t)$ is the neutrino conversion probability $P_{\nu_{a} \rightarrow \nu_{b}}(t)$ and $H_{d}=H_{a a}(t)-H_{b b}(t)$ and dot on the top corresponds to derivative with respect to $t$. Using the Eq.(2) in Eq.(3) we can write

$$
\begin{aligned}
\dot{P}(t) & =-2 H_{a b}(t) \int_{0}^{t} \dot{I}\left(t_{1}\right) d t_{1} \\
& =-2 H_{a b}(t) \int_{0}^{t}\left[H_{a b}\left(t_{1}\right)\left(2 P\left(t_{1}\right)-1\right)+H_{d}\left(t_{1}\right) R\left(t_{1}\right)\right] d t_{1} .
\end{aligned}
$$

Let us consider the neutrino propagation in the medium in the presence of a magnetic field. Then we have

$$
H_{d}(t)=V-\Delta \cos 2 \theta+V_{a x i a l} \quad \text { and } \quad H_{a b}(t)=\mu B_{\perp}(t)
$$


The quantity $V$ is the difference of neutrino vector potential for $\nu_{a} \rightarrow \nu_{b}, \Delta=\left(m_{2}^{2}-m_{1}^{2}\right) / 2 E=\Delta m^{2} / 2 E$ and $E$ is the neutrino energy and $\theta$ is the neutrino mixing angle. The axial vector potential $V_{\text {axial }}=\mu_{e f f} \mathbf{k} . \mathbf{B}(t) / k$ is generated by the mean axial vector current of charged leptons in an external magnetic field [11 13, 16]. For fluctuation in the magnetic field we can write $B(t)=B_{0}+B^{\prime}(t)$, where $B_{0}$ is the constant background field and $B^{\prime}(t)$ is the random fluctuation over it. Then we have

$$
\begin{aligned}
H_{d}(t) & =H_{d}(0)+H_{d}^{\prime}(t) \\
& =\left(V-\Delta+\mu_{e f f} B_{\| 0}\right)+\mu_{e f f} B_{\|}^{\prime}(t),
\end{aligned}
$$

and

$$
\begin{aligned}
H_{a b}(t) & =H_{a b}(0)+H_{a b}^{\prime}(t) \\
& =\mu B_{\perp 0}+\mu B_{\perp}^{\prime}(t) .
\end{aligned}
$$

For the neutrino conversion length greater than the domain size i.e. $l_{\text {conv }}>>L_{0}$ (where $l_{\text {conv }} \sim 1 / \mathcal{P} \Gamma_{W}$ and $\Gamma_{W}$ is the weak interaction rate), a neutrino will cross many magnetic field domains before it flips its helicity. Thus the neutrino will experience an average field before it flips its helicity. So one can average the propagation equation (4) over the random magnetic field distribution [9 [17]. The magnetic field in different domains is randomly oriented with respect to the neutrino propagation direction. So the neutrino conversion probability depends on the root mean square $(\mathrm{rms})$ value of the random magnetic field. With the use of the delta correlation for uncorrelated magnetic field domains of size $L_{0}$, the average of the random magnetic field is $13,17,20$,

$$
\begin{gathered}
\left\langle B_{\|}(t)\right\rangle=\left\langle B_{\perp}(t)\right\rangle=\left\langle B_{\|}(t) B_{\perp}(t)\right\rangle=0, \\
\left\langle B_{i \|}(t) B_{j \|}\left(t_{1}\right)\right\rangle=\left\langle B_{\|}^{2}\right\rangle \delta_{i j} L_{0} \delta\left(t-t_{1}\right),
\end{gathered}
$$

and

$$
\left\langle B_{i \perp}(t) B_{j \perp}\left(t_{1}\right)\right\rangle=\left\langle B_{\perp}^{2}\right\rangle \delta_{i j} L_{0} \delta\left(t-t_{1}\right) .
$$

The $r m s$ value of the averaged magnetic field is given as $B_{r m s}=\sqrt{\left\langle B^{2}\right\rangle}$. Let us assume,

- $H_{a b}(t)$ is sufficiently close to $H_{a b}\left(t_{2}\right)$ so that

$$
\int_{0}^{t} \int_{0}^{t_{1}} H_{a b}(t) \frac{H_{d}\left(t_{1}\right) H_{d}\left(t_{2}\right)}{H_{a b}\left(t_{2}\right)} \dot{P}(t) d t_{2} d t_{1} \simeq \int_{0}^{t} \int_{0}^{t_{1}} H_{d}\left(t_{1}\right) H_{d}\left(t_{2}\right) \dot{P}(t) d t_{2} d t_{1},
$$

and

- $P(t)$ has no correlation with $H_{d}(t)$ and $H_{a b}(t)$.

Thus averaging both sides of Eq.(体) and using the above magnetic field correlations we obtain

$$
\left.\dot{\mathcal{P}}(t)=-2 H_{a b}^{2}(0)\left\langle\int_{0}^{t}\left(2 P\left(t_{1}\right)-1\right) d t_{1}\right\rangle-\Gamma_{\perp}\langle 2 P(t)-1)\right\rangle-H_{d}^{2}(0)\left\langle\int_{0}^{t} P\left(t_{1}\right) d t_{1}\right\rangle-2 \Gamma_{\|}\left\langle\int_{0}^{t} \dot{P}\left(t_{1}\right) d t_{1}\right\rangle .
$$


where $\langle P\rangle=\mathcal{P}$ and the quantities $\Gamma_{\perp}$ and $\Gamma_{\|}$are the longitudinal and transverse damping parameters given by

$$
\Gamma_{\perp}=\frac{4}{3} \mu^{2}\left\langle B^{2}\right\rangle L_{0}, \quad \text { and } \quad \Gamma_{\|}=\frac{1}{6} \mu_{e f f}^{2}\left\langle B^{2}\right\rangle L_{0} .
$$

Differentiating once again to Eq.(12) with respect to $t$ we obtain the following second order differential equation

$$
\ddot{\mathcal{P}}(t)+2 \Gamma_{T} \dot{\mathcal{P}}(t)+\omega_{s}^{2} \mathcal{P}(t)-2 H_{a b}^{2}(0)=0,
$$

where the quantity

$$
\omega_{s}^{2}=4 H_{a b}^{2}(0)+H_{d}^{2}(0)
$$

is square of the spin rotation frequency of the neutrino in the medium, $\Gamma_{T}=\Gamma_{\perp}+\Gamma_{\|}$and it satisfies the boundary conditions $\mathcal{P}(0)=0$ and $\dot{\mathcal{P}}(0)=\Gamma_{\perp}$. The solution to this differential equation is given by,

$$
\mathcal{P}(t)=\frac{2 H_{a b}^{2}(0)}{\omega_{s}^{2}}\left(1-e^{-\Gamma_{T} t}\left\{\frac{\sinh \left[\sqrt{\Gamma_{T}^{2}-\omega_{s}^{2}} t\right]}{\sqrt{\Gamma_{T}^{2}-\omega_{s}^{2}}}\left[\Gamma_{\|}-\frac{\Gamma_{\perp}\left(\omega_{s}^{2}-2 H_{a b}^{2}(0)\right)}{2 H_{a b}^{2}(0)}\right]+\cosh \left[\sqrt{\Gamma_{T}^{2}-\omega_{s}^{2}} t\right]\right\}\right) .
$$

The Eq.(16) is the general solution for the average conversion probability having random fluctuation in both transverse and longitudinal mode of the magnetic field [13]. For no random fluctuation in the magnetic field $\left(\Gamma_{\perp}=\Gamma_{\|}=0\right)$ we get back the standard MSW type solution for the neutrino conversion probability

$$
P(t)=\frac{4 H_{a b}^{2}(0)}{\omega_{s}^{2}} \sin ^{2}\left(\frac{\omega_{s} t}{2}\right) .
$$

Let us consider the propagation of a system of active (doublet) and light sterile (singlet) neutrinos $\left(\nu_{e} \rightarrow \nu_{s}\right)$, with masses $m_{1}$ and $m_{2}$, mixing angle $\theta$, and no transition magnetic moments, in the presence of a magnetised plasma. Then the Hamiltonian in the evaluation equation Eq.(1) will be

$$
\left(\begin{array}{cc}
V-\Delta \cos 2 \theta+\mu_{e f f} \mathbf{k} \cdot \mathbf{B} / k & \Delta \sin 2 \theta / 2 \\
\Delta \sin 2 \theta / 2 & 0
\end{array}\right),
$$

where $\Delta=\left(m_{2}^{2}-m_{1}^{2}\right) / 2 E$. For active-sterile neutrino conversion the resultant vector potential experienced by $\nu_{e}$ is given by

$$
V=\sqrt{2} G_{F} n_{e}\left(3 Y_{e}+4 Y_{\nu_{e}}-1\right)
$$

where $G_{F}$ is the Fermi coupling constant, $n_{e}$ is the electron density in the medium and $Y_{e}$ and $Y_{\nu_{e}}$ are the electron and $\nu_{e}$ abundances respectively in the medium. For neutrino propagating along the $z$ axis the $V_{\text {axial }}$ is

$$
V_{\text {axial }}=\mu_{e f f} B_{z} \frac{k_{z}}{k}
$$

The quantity $\mu_{e f f}$ for $\nu_{e} \rightarrow \nu_{s}$ is given by

$$
\mu_{e f f}=\frac{e G_{F} P_{F}}{\sqrt{2} 2 \pi^{2}}
$$


and $P_{F}$ is the Fermi momentum of electron. As we are considering the neutrino magnetic moment/transition magnetic moment to be zero, the perpendicular component of the damping term $\Gamma_{\perp}$ will vanish. Thus putting $\Gamma_{\perp}=0$ in Eq.(16), the average conversion probability for $\nu_{a} \rightarrow \nu_{s}$ will be

$$
\mathcal{P}(t)=\frac{\Delta^{2} \sin ^{2} 2 \theta}{2 \omega_{f}^{2}}\left(1-e^{-\Gamma_{\|} t}\left\{\Gamma_{\|} \frac{\sinh \left[\sqrt{\Gamma_{\|}^{2}-\omega_{f}^{2}} t\right]}{\sqrt{\Gamma_{\|}^{2}-\omega_{f}^{2}}}+\cosh \left[\sqrt{\Gamma_{\|}^{2}-\omega_{f}^{2}} t\right]\right\}\right),
$$

where

$$
\omega_{f}^{2}=\left(H_{d}^{2}(0)+\Delta^{2} \sin ^{2} 2 \theta\right)
$$

is square of the flavor conversion frequency of the neutrino in the medium. In a supernova medium with a strong random magnetic field satisfying the condition $\Gamma_{\|} \gg \omega_{f}$ the average conversion probability in Eq.(22) will be approximately

$$
\mathcal{P}(t) \simeq \frac{\Delta^{2} \sin ^{2} 2 \theta}{2 \omega_{f}^{2}}\left(1-e^{-\omega_{f}^{2} t / 2 \Gamma_{\|}}\right)
$$

and for weak field limit i.e. $\Gamma_{\|} \ll \omega_{f}$ it will be

$$
\mathcal{P}(t) \simeq \frac{\Delta^{2} \sin ^{2} 2 \theta}{2 \omega_{f}^{2}}\left(1-e^{-\Gamma_{\|} t} \cos \omega_{f} t\right)
$$

These are shown previously by Semikoz and Valle in ref [13]. Using the strong field limit for the active-sterile neutrino conversion in a supernova, the limit on $\Delta m^{2}$ and $\sin ^{2} 2 \theta$ are obtained from the supernova cooling [10] and by thermalisation of the sterile neutrinos in the early universe hot plasma [13].

\section{B. method - II}

In the previous section we have derived the master equation for the average conversion probability of neutrino in the medium with a magnetic field. But in that calculation we have neglected the correlation of $P(t)$ with $H_{d}(t)$ and $H_{a b}(t)$. In the previous section we have only taken the average of the equation for $\dot{P}(t)$ and not for $\dot{R}(t)$ and $\dot{I}(t)$ to derive the master equation for the conversion probability. For the neutrino conversion length greater than the domain size $\left(l_{\text {conv }}>>L_{0}\right)$, a neutrino will cross many magnetic field domains before it flips its helicity. Thus the neutrino will experience an average field before it flips its helicity. So one can average the propagation equations (2) and (3) over the random magnetic field distribution [9, 18]. Let us define the average of the functions $\langle P(t)\rangle=\mathcal{P}(t),\langle R(t)\rangle=\mathcal{R}(t)$ and $\langle I(t)\rangle=\mathcal{I}(t)$. Because of the averaging the average probability $\mathcal{P}(t)$ will only depend on the even powers of the magnetic field correlation. Using the average functions in Eqs.(8) to (10) we obtain

$$
\begin{aligned}
\dot{\mathcal{I}}(t)= & \left\langle H_{a b}(t)(2 P(t)-1)\right\rangle+\left\langle H_{d}(t) R(t)\right\rangle \\
= & H_{a b}(0)\langle(2 P(t)-1)\rangle+\left\langle\tilde{H}_{a b}(2 P(t)-1)\right\rangle \\
& +H_{a a}(0)\langle R(t)\rangle+\left\langle\tilde{H}_{a a}(t) R(t)\right\rangle,
\end{aligned}
$$

and 


$$
\dot{\mathcal{R}}(t)=-H_{a a}(0)\langle I(t)\rangle-\left\langle\tilde{H}_{a a}(t) I(t)\right\rangle
$$

respectively. Using the delta correlations for the magnetic fields as shown in Eqs.(8) to (10) we obtain

$$
\left\langle H_{d}^{\prime}(t) R(t)\right\rangle \simeq-2 \Gamma_{\|} \mathcal{I}(t) ; \quad\left\langle H_{a b}^{\prime}(t) P(t)\right\rangle \simeq-\Gamma_{\perp} \mathcal{I}(t),
$$

and

$$
\left\langle H_{a b}^{\prime}(t) I(t)\right\rangle \simeq \frac{\Gamma_{\perp}}{2}(2 \mathcal{P}(t)-1) .
$$

Putting these values in Eqs.(26) and (27) we obtain

$$
\dot{\mathcal{I}}(t)=H_{d}(0) \mathcal{R}(t)+H_{a b}(0)(2 \mathcal{P}(t)-1)-2\left(\Gamma_{\perp}+\Gamma_{\|}\right) \mathcal{I}(t) .
$$

and

$$
\dot{\mathcal{R}}(t)=-H_{d}(0) \mathcal{I}(t)-2 \Gamma_{\|} \mathcal{R}(t) .
$$

Using these equations in Eq.(3) we obtain

$$
\dot{\mathcal{P}}(t)=-2 H_{a b}(0) \mathcal{I}(t)-\Gamma_{\perp}(2 \mathcal{P}(t)-1) .
$$

For convenience let us define

$$
\mathcal{I}(t)=e^{-2\left(\Gamma_{\|}+\Gamma_{\perp}\right) t} \mathcal{I}_{1}(t),
$$

and

$$
\mathcal{R}(t)=e^{-2 \Gamma_{\|} t} \mathcal{R}_{1}(t),
$$

Using Eqs.(33) and (34) in Eqs.(30) to (32) we obtain

$$
\begin{gathered}
\dot{\mathcal{I}}_{1}(t)=H_{d}(0) e^{2 \Gamma_{\perp} t} \mathcal{R}_{1}(t)+H_{a b}(0) e^{2\left(\Gamma_{\perp}+\Gamma_{\|}\right) t}(2 \mathcal{P}(t)-1), \\
\dot{\mathcal{R}}_{1}(t)=-H_{d}(0) e^{-2 \Gamma_{\perp} t} \mathcal{I}_{1}(t)
\end{gathered}
$$

and

$$
\dot{\mathcal{P}}(t)=-2 H_{a b}(0) e^{-2\left(\Gamma_{\perp}+\Gamma_{\|}\right) t} \mathcal{I}_{1}(t)-\Gamma_{\perp}(2 \mathcal{P}(t)-1) .
$$

Differentiating Eq.(37) twice with respect to $t$ and putting the value of $\dot{\mathcal{I}}_{1}(t)$ from Eq.(35) we obtain the master equation for the average conversion probability as,

$$
\begin{aligned}
\dddot{\mathcal{P}}(t) & +4\left(\Gamma_{\perp}+\Gamma_{\|}\right) \ddot{\mathcal{P}}(t)+4\left(3 \Gamma_{\perp} \Gamma_{\|}+\Gamma_{\perp}^{2}+\Gamma_{\|}^{2}+\frac{\omega_{s}^{2}}{4}\right) \dot{\mathcal{P}}(t) \\
& +8\left(\Gamma_{\perp}^{2} \Gamma_{\|}+\Gamma_{\perp} \Gamma_{\|}^{2}+H_{a b}^{2}(0) \Gamma_{\|}+\frac{H_{d}^{2}(0) \Gamma_{\perp}}{4}\right) \mathcal{P}(t) \\
& -4\left(\Gamma_{\perp}^{2} \Gamma_{\|}+\Gamma_{\perp} \Gamma_{\|}^{2}+H_{a b}^{2}(0) \Gamma_{\|}+\frac{H_{d}^{2}(0) \Gamma_{\perp}}{4}\right)=0
\end{aligned}
$$


with the boundary conditions $\mathcal{P}(0)=0, \quad \dot{\mathcal{P}}(0)=\Gamma_{\perp}$ and $\ddot{\mathcal{P}}(0)=2 H_{a b}^{2}(0)-2 \Gamma_{\perp}^{2}$. By switching off the damping terms in Eq. (38), we will give get back the same solution for $\mathrm{P}(\mathrm{t})$ as shown in Eq.(17). The solution to the above third order differential equation is given in Eq.(25) of the ref [19] and it is of the form

$$
\mathcal{P}(t)=\frac{1}{2}+y(t) .
$$

Henceforth for further reference we will refer the Eq.(25) of ref [19]. The solution obtained is very complicated and it is difficult to conclude any thing from the solution. On the other hand the interesting part of the solution is that, the positive definiteness of the average conversion probability $(0 \leq \mathcal{P} \leq 1)$ requires the following condition to satisfy

$$
\omega_{s}^{2}>\frac{4}{3}\left(\Gamma_{\perp}^{2}+\Gamma_{\|}^{2}-\Gamma_{\perp} \Gamma_{\|}\right)
$$

irrespective of the form of neutrino potential and the magnetic field, which essentially shows that neutrino crosses many domains leads to this requirement. Considering the magnetic field in the early universe hot plasma and the core of the newly born neutron stars to be purely random in nature, we studied the conversion $\nu_{e L} \rightarrow \nu_{e R}$ in these medium in a previous paper [19].

\section{COMPARISON OF BOTH THE METHODS}

Let us consider the active sterile neutrino conversion in the magnetised medium, with neutrino mixing and $\mu=0\left(\Gamma_{\perp}=0\right)$ as shown in (18). Only the fluctuation in the parallel component of the magnetic field will contribute. So for this case the condition in Eq.(40) will be modified to

$$
\omega_{f}^{2}>\frac{4 \Gamma_{\|}^{2}}{3} .
$$

This implies $\omega_{f}>\Gamma_{\|}$. The average conversion probability in Eq.(24) for strong random magnetic field limit is derived with the approximation $\Gamma_{\|} \gg \omega_{f}$. On the other hand we obtain from Eq.(41), completely the opposite one. Also the later condition further shows that, what ever may be the strength of the random component of the magnetic field $\Gamma_{\|}$, it should always satisfy the condition in Eq.(41). Apart from that, this condition comes automatically from the positive definiteness of the probability. This opposite situation arises because of the averaging procedure. In the first case we only take the average of the Eq.(3) and use the functions $I(t)$ and $R(t)$ in that. On the other hand in the second method, we average the equations for $P(t), I(t)$ and $R(t)$ separately, which are shown explicitly. In the first method it is assumed that $H_{a b}(t)$ is sufficiently close to $H_{a b}\left(t_{2}\right)$ so that in Eq.(11), they cancel. But in general this is not true for arbitrary $t$ and $t_{2}$. Such terms are not there in the second method to derive the average probability equation.

Comparison of both the master equations Eq.(14) and Eq.(38) shows that, in the first there is no mixing between the longitudinal $\Gamma_{\|}$and transverse $\Gamma_{\perp}$ damping terms. Also the damping terms do not mix with

the background terms. On the other hand, there is mixing between the damping and background terms, and the probability equation becomes more complicated. 


\section{RESULTS AND DISCUSSIONS}

Let us estimate the range of $\Delta m^{2}$ and $\sin ^{2} 2 \theta$ from the the above inequality in Eq.(41) in a supernova environment. Inside the supernova the vector potential for the process $\nu_{e} \rightarrow \nu_{s}$ is

$$
V \simeq 4 \times 10^{-6} \rho_{14}\left(3 Y_{e}+4 Y_{\nu_{e}}-1\right) M e V \simeq 4.48 \mathrm{eV},
$$

where $Y_{e} \simeq 0.3, Y_{\nu_{e}} \simeq 0.06$ and the quantity $\rho_{14} \simeq 8$ is the density in the supernova core in units of $10^{14} \mathrm{gm} / \mathrm{cm}^{3}$. Now let us assume that the random fluctuation in the magnetic field is much large compared to the constant background part i.e. $\mu B_{\| 0} \ll \Gamma_{\|}$as considered in ref [10]. The flavor precession frequency for $\nu_{e} \rightarrow \nu_{s}$ is given by

$$
\omega_{f}=\sqrt{\left(V-\frac{\Delta m^{2}}{2 E} \cos 2 \theta\right)^{2}+\left(\frac{\Delta m^{2}}{2 E}\right)^{2} \sin ^{2} 2 \theta} .
$$

Inside the supernova core, neutrinos have energy in the range 30 to $100 \mathrm{MeV}$. Then considering $E \simeq 100$ $\mathrm{MeV}$, we obtain

$$
\omega_{f}=\sqrt{\left(4.48-0.5 \times 10^{-8} \frac{\Delta m^{2}}{e V^{2}} \cos 2 \theta\right)^{2}+0.25 \times 10^{-16}\left(\frac{\Delta m^{2}}{e V^{2}}\right)^{2} \sin ^{2} 2 \theta} \mathrm{eV} .
$$

Inside the supernova core for $\nu_{e} \rightarrow \nu_{s}$

$$
\mu_{e f f} \simeq 4.3 \times 10^{-13} \mu_{B}\left(\frac{P_{F}}{M e V}\right),
$$

where $\mu_{B}$ is the Bohr magneton. The quantity $\mu_{e f f}$ has the same dimension that of the magnetic moment, but it has nothing to do with the magnetic moment as it does not change the helicity of the particle. The Fermi momentum of the electron inside the core is

$$
P_{F} \simeq 320\left(Y_{e} \rho_{14}\right)^{1 / 3} \quad M e V \simeq 428 \mathrm{MeV} .
$$

Thomson and Dunkan have argued that very strong magnetic fields might be generated inside the supernova core due to small scale dynamo mechanism. If these fields are generated after core collapse, then it could be viewed as random superposition of many small dipoles of size $L_{0} \sim 1 \mathrm{Km}$. Then the longitudinal damping parameter is simplified to

$$
\Gamma_{\|} \simeq 9.6 B_{14}^{2} \mathrm{eV}
$$

where $B_{r m s}$ is expressed in units of $10^{14}$ Gauss. Putting $\omega_{f}$ and $\Gamma_{\|}$in Eq.(41), we can find the ranges of $\Delta m^{2}$ and $\sin ^{2} 2 \theta$ for which the condition in Eq.(41) is satisfied. These are shown in the contour plots for different values of the magnetic fields in Figure 1. Figure 1 (a) shows that, a very narrow range of $\Delta m^{2} / e V^{2}\left(3.16 \times 10^{8}-1.6 \times 10^{9}\right)$ is excluded for $0 \lesssim \sin ^{2} 2 \theta 0.63 \times 10^{-3}$ for the random magnetic field strength $B_{r m s}=0.1 \times 10^{14}$ Gauss. By increasing the strength of the random magnetic field to $0.5 \times 10^{14}$ and $0.63 \times 10^{14}$ Gauss, we see in Figure 1 (b) and (c) that the width of the excluded range of $\Delta m^{2} / e V^{2}$ has increased. The spread of $\Delta \mathrm{m}^{2} / \mathrm{eV}^{2}$ towards smaller values is large compared to the large values. Also the large values of $\sin ^{2} 2 \theta$ are excluded. In Figure 1 (b) we see that $3.5 \times 10^{8} \lesssim \Delta m^{2} / e V^{2} \lesssim 1.6 \times 10^{9}$ is excluded 
and for this the excluded range of $\sin ^{2} 2 \theta$ is $0 \lesssim \sin ^{2} 2 \theta \lesssim 0.4$ (for $B_{r m s}=0.5 \times 10^{14}$ Gauss). In Figure 1 (c) it is shown that for $B_{r m s}=0.63 \times 10^{14}$ Gauss, the excluded region of $\Delta m^{2} / e V^{2}$ is $1.6 \times 10^{7}-1.6 \times 10^{9}$ and in this range all the values of $\sin ^{2} 2 \theta$ are excluded. It shows that, two distinct allowed regions for $\Delta m^{2} / e V^{2}$ are there in both sides of the excluded region. In these allowed regions all ranges of $\sin ^{2} 2 \theta$ are allowed. But so far as $\Delta m^{2}$ value is concerned, it is interesting to consider only the region left to the excluded curve, because it corresponds to smaller $\Delta m^{2} / e V^{2}$ values $\left(\Delta m^{2} \lesssim(k e V)^{2}\right)$. Going from Figure 1 (c) to (d) (for

$B_{r m s}=10^{14}$ Gauss) we observe that, the left arm of the curve vanishes and the right arm spreads towards the higher values of $\Delta m^{2} / e V^{2}$ for all ranges of $\sin ^{2} 2 \theta$. This implies that small values of $\Delta m^{2} / e V^{2}$ are excluded. By further increasing the strength of the random magnetic field, we have shown in Figure 1 (e) that, (for $B_{r m s}=10^{16}$ Gauss) all values of $\Delta m^{2} / e V^{2} \lesssim 1.6 \times 10^{13}$ are excluded for all ranges of $\sin ^{2} 2 \theta$ values. This range of $\Delta \mathrm{m}^{2} / \mathrm{eV}^{2}$ are obviously not at all interesting, because it corresponds to very high values of $\Delta \mathrm{m}^{2} / \mathrm{eV}^{2}$, and no astrophysical, cosmological and laboratory observations favour this. Also if we consider small values of magnetic field $\left(B_{r m s}<10^{13}\right.$ Gauss) then we found that, the excluded region vanishes and all the parameter ranges are allowed. Thus if we consider only the effect of random magnetic field in a magnetised electron plasma along the neutrino propagation direction, then magnetic field should satisfy $B_{r m s} \lesssim 10^{14}$ Gauss so that small $\Delta m^{2} / \mathrm{eV}^{2}$ ranges should not be excluded. On the other hand for $B_{r m s}<10^{13}$ Gauss we found that, all the parameter ranges are allowed. As we have shown in Figure 1 (a), only a very narrow strip of $\Delta m^{2} / e V^{2}$ values are excluded for $0 \lesssim \sin ^{2} 2 \theta \lesssim 0.4$ and this narrow strip vanishes for smaller values of $B_{r m s}$. For $\Delta m^{2}$ and $\sin ^{2} 2 \theta$ very small, we can see in Eq.(44) that $\omega_{f} \simeq 2.12$ $\mathrm{eV}$ and the condition Eq.(41) will give $B_{r m s}<0.4 \times 10^{14}$ Gauss. Thus we found that for magnetic field $B_{r m s} \lesssim .63 \times 10^{14}$ Gauss, all the interesting ranges of parameters are allowed. Also this implies that the maximum value of $\Delta m^{2}$ can be in the $(k e V)^{2}$ range.

\section{CONCLUSION}

For neutrino propagating in the magnetised plasma of the supernova, will experience an axial potential which is proportional to the scalar product of the neutrino momentum and the magnetic field. We derived the average conversion probability for active neutrino goes to active or sterile one by using a method developed by Semikoz et. al, and another by one of us where it is assumed that the magnetic field has a random fluctuation over the constant background. In the second method for neutrino having non-zero mixing and zero magnetic moment we found the condition for the positive definiteness of the average conversion probability for the process $\nu_{e} \rightarrow \nu_{s}$. Using this condition as the basis, we found the excluded/included ranges of $\Delta m^{2}$ and $\sin ^{2} 2 \theta$ for different values of the magnetic field. Our calculation shows that for neutrino to have maximum mass in the $\mathrm{keV}$ range or less, the random magnetic field along the neutrino propagation direction should not be very large $\left(B_{r m s} \lesssim 0.63 \times 10^{14}\right.$ Gauss). Because it is observed that for large magnetic field, small values of $\Delta m^{2}$ are excluded. 
(a)

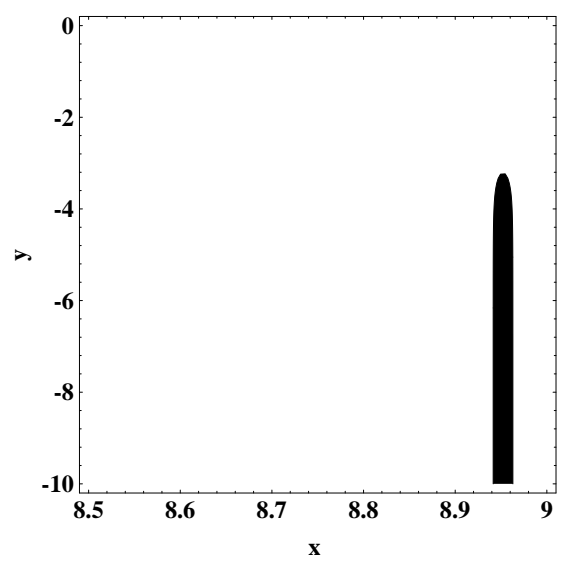

(c)

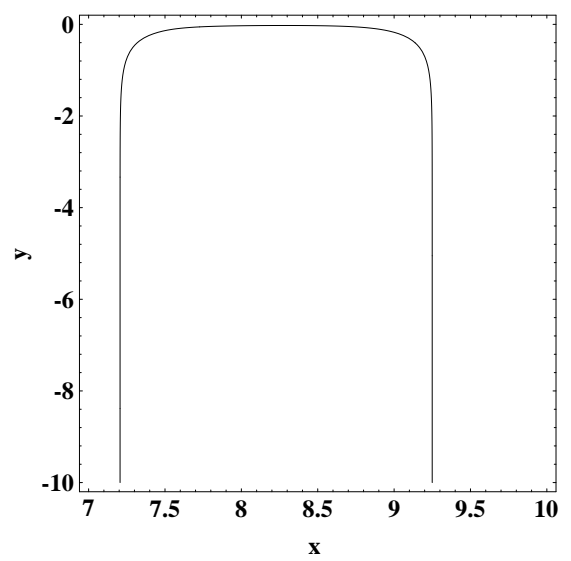

(b)

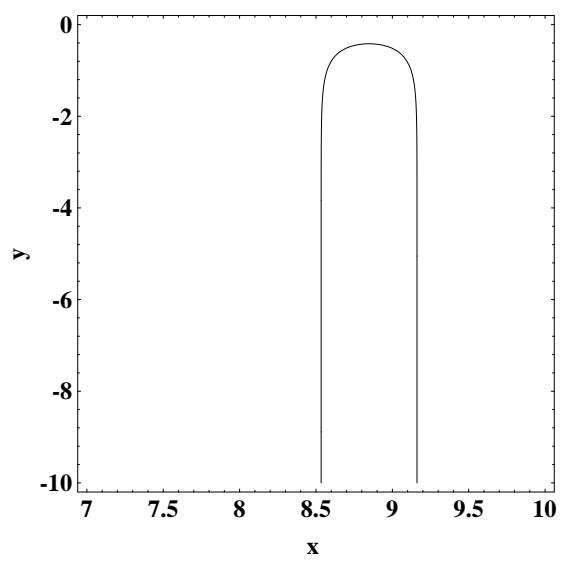

(d)

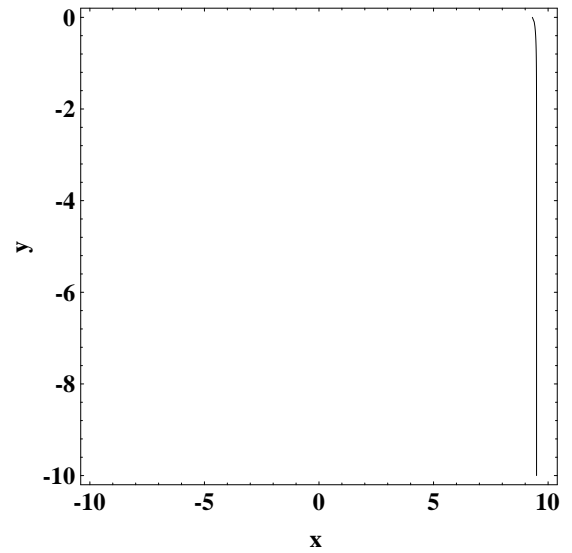

(e)

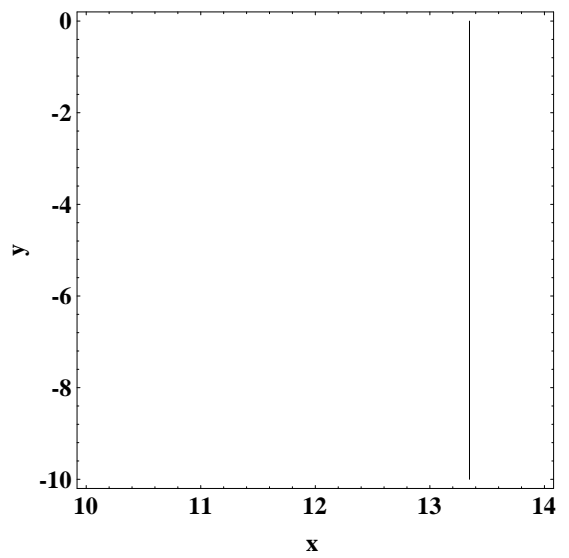

Figure 1: We have defined $\mathbf{x}=\log \left[\Delta m^{2} / e V^{2}\right]$ and $\mathbf{y}=\log \left[\sin ^{2} 2 \theta\right]$.(a) It is for $B_{r m s}=10^{13}$ Gauss and the shaded region is excluded, (b) for $B_{r m s}=0.5 \times 10^{14}$ Gauss and the region inside the curve is excluded, (c) $B_{r m s}=0.63 \times 10^{14}$ Gauss and the region inside the curve is excluded, (d) for $B_{r m s}=10^{14}$ Gauss and left side of the curve is excluded and (e) $B_{r m s}=10^{16}$ Gauss and the left side of the curve is excluded. 
* mail: sarira@prl.ernet.in and sarira@plasma.ernet.in

[1] G. F.Smoot et. al., Astrophys. J. 396, L1 (1992).

[2] E. L. Wright et. al., Astrophys. J. 396, L13 (1992); M. Davis, F. J. Summers and D. Schagel, Nature 359, 393 (1992); A. N. Taylor and M. Rowan-Robinson, ibid 359, 396 (1992); R. K. Schaefer and Q. Shafi, Nature 359, 199 (1992); J. A. Holtzman and J. R. Primack, Astrophys. J. 405, 428 (1993) and A. Klypin et. al., Astrophys. J. 416, 1 (1993).

[3] J. T. Peltoniemi, D. Tommasini and J. W. F. Valle, Phys. Lett. B 298, 383 (1993).

[4] E. J. Chun, A. S. Joshipura and A. Yu. Smirnov, Phys. Lett. B 357, 608 (1995); E. J. Chun, A. S. Joshipura and A. Yu. Smirnov, Phys. Rev. D 54, 4654 (1996).

[5] J. T. Peltoniemi and J. W. F. Valle, Nucl. Phys. B 406, 409 (1993); D. O. Caldwell and R. N. Mohapatra, Phys. Rev. D 48, 3259 (1993).

[6] E. Akhmedov, Z. Berezhiani, G. Senjanovic and Z. Tao, Phys. Rev. D 47, 3245 (1993).

[7] T. Walker, G. Steigman, D. N. Schramm, K. Olive and H. Kang, Astrophys. J. 376, 51 (1991).

[8] Ya. Zeldovich, A. A. Ruzmaikin and D. D. Sokoloff, Magnetic Fields in Astrophysics, Mc Graw Hill, $1983 ;$ E. Parker, Cosmological Magnetic Fields, Oxford Univ. Press, 1979; Peter Meszaros, High Energy Radiation from Magnetised Neutron Stars, Chicago Univ. Press, 1992.

[9] S. Pastor, V. Semikoz and J.W.F. Valle, Phys. Lett. B369, 301 (1996).

[10] S. Pastor, V. Semikoz and J.W.F.Valle, Astroparticle Phys. 3, 87 (1995).

[11] S. Sahu, V. Semikoz and J. W. F. Valle, hep-ph/9512390.

[12] J. C. D'Olivo and J. Nieves, Phys. Lett. B 383, 87 (1995).

[13] V. Semikoz and J. W. F. Valle, Nucl. Phys. B425, 651 (1994); V. Semikoz and J. W. F. Valle, hep-ph/9607208.

[14] H. Nunokawa, V. B. Semikoz, A. Yu. Smirnov and J. W. F. Valle, Nucl. Phys. B 501, 17 (1997).

[15] A. Kuseno and G. Segre, Phys. Rev. Lett. 77, 4872 (1996).

[16] S. Esposito and G. Capone, Z. Phys. C70, 55 (1996).

[17] K. Enqvist, A. Rez and V. Semikoz, Nucl. Phys. B436, 49 (1995).

[18] K. Enqvist, J. Maalampi and V. B. Semikoz, Nucl. Phys. B456, 339 (1995).

[19] S. Sahu, Phys. Rev. D 56, 4378 (1997).

[20] F. N. Loreti and A. B. Balantekin, Phys. Rev. D50, 4762 (1994); A. B. Balantekin, J. M. Fetter and F. N. Loreti Phys. Rev. D54 3941 (1996). 